The Chittagong Univ. J. B. Sci., Vol. 6(1 \&2):107-118, 2011.

\title{
ATTITUDE TOWARD EVE TEASING
}

\author{
BIPLOB KUMAR DEY ${ }^{1}$, ARUNAVO BAIRAGI ${ }^{1}$ AND AYESHA MAHMUDA ${ }^{2}$ \\ ${ }^{1}$ Department of Psychology, University of Chittagong, Chittagong-4331, Bangladesh. \\ ${ }^{2}$ Associate Professor, Department of Psychology, University of Dhaka, Dhaka- 1000, Bangladesh.
}

\begin{abstract}
The present study was conducted to measure the attitude toward eve teasing. For this purpose a scale was developed in Likert format. The scale consisted of 10 statements in Bangla. Based on preliminary scrutinizing and expert judgment for face validity, 29 statements were administered to 100 males and 100 females for items analysis. Of the total 29 statements 10 were found to have significant item total correlation $(\alpha=.01$ to 0.05$)$ and were retained in the final scale. For measuring reliability of this split half 0.52 and chronbach's alpha 0.56 were computed. This scale was then administered to 200 people (100 males and 100 females) living in different areas of Dhaka city. The obtained data were analyzed by using ANOVA. The data revealed that the interaction effect of gender levels and education was found significant.
\end{abstract}

Key words: Attitude, eve teasing,

\section{INTRODUCTION}

Violence against women has become one of the most visible and articulate social problems around the world. It is a critical human rights and serious development issue. It is a universal phenomenon but takes different forms in different socio-cultural and religious contexts. Eve teasing is one of the most serious forms of violence against women by men in more or less all over the world. Eve teasing means when some young boys or men are throwing speech toward a girl or women such as rude comments, touching, groping, stalking, sending text messages, prank calls, heartening and intimidation, acid attacks, ogling, walking, obscene song, whistle, rude posture, proposing love or offering marriage, giving letter, threatening etc. these activities are called eve teasing.

In Bangladesh, eve teasing is a widespread and most disturbing reality for the girls. In Bangladesh, various reports and research findings show that at least several dozens of girls committed suicide in the last five years. They did so to get a respite from incessant teasing and bullying by males. Eve teasing in the college or university campus initiated by students is most common, teacher or other

*Corresponding author:biplob480@yahoo.com 
employees can also perpetrate it, and the victim can be a girl, a female teacher, or other employees. Because of the strict patriarchal social system, it is almost daring a woman to protect against teasing in the university campus.

Eve teasing is a euphemism used in India, Bangladesh and Pakistan for public sexual harassment, street harassment or molestation of women by men. The term of 'eve teasing' has different usages in different countries. In France reference is made to 'droit de cuissage' (right to the thigh), in India the term 'eve teasing' has been used, in the Netherland it is referred to as 'unsolicited or undesired intimacies', while in Malaysia words like 'gatal or miang' (literally means itchy) are often used to described it. However, the condition of "eve teasing" is so acute in India. (Puri 1999)

The term 'Eve-teasing' most likely references the biblical 'Eve', the supposed first woman whose appearance seems to fair, divinely fair, beauty, fairest of creation and the quotations of 'Teasing' comes from an animal whose mouth fills with venom. Eve teasing means harassment of or sexually aggressive behavior toward women. The concept of eve teasing vary among and within societies, depending on whether individual are born and socialized as men or women in a specific social economic class in a society, universal consensus exists on the key characteristics of definition on eve teasing or sexual harassment (Berret 2006). According to CEC (1993), "Sexual harassment or eve teasing means unwanted conduct of a sexual nature, or other conduct based on sex, affecting the dignity of men and women in the society. This can include unwelcome physical, verbal, or non verbal conduct."

There are many forms of eve teasing, which are power-player, intellectual seducer, mother-father, one-of-the-gang, unintentional and the eve-teaser are conducting eve teasing toward girls by these forms. Therefore, eve-teaser may be anyone, such a client, a co-worker, a teacher or professor, a student, a friend, or a stranger and eve-teasing may occur any place such as beaches, roads, cinema halls, buses and sadly even in educational institutions. It is a crime easy to commit but difficult to prove. Any kinds of sexual behavior, showing pornography, proposed sexual relationship or showing and conducting in these types of activities are considered sexual harassment. Too much chapter reviews some existing literature on eve teasing done in Bangladesh context by different researchers. Review the literature would help us to provide with a clear picture of eve teasing. Shampa (2003) Stated that, sexual harassment at public is a new term 
to describe the old age problem of unwelcome conduct of a sexual nature. In our society tragedies like Simi, Mahima, Idrani, Fahima, Rumi, and many others continues because here perpetrators and culprits recklessly harass the young girls irrespective of the law enforcers, which give them free field and impose restrictions and constraints upon the normal life of girls that something make them feel their lives unbearable.

According to Huda (1999) Eve teasing or sexual harassment is widely prevalent in a different forms, and women in every spheres of life are to some extent, whether overtly or covertly, exposed to such persecution. However, this issues has not, until recently been addressed with any great seriousness in Bangladesh. A small empirical investigation on sexual harassment was conducted on 78 randomly selected respondents. Respondents included government servants, students, NGO workers, domestics' servants. Approximately 53\% answered in affirmative when they were asked whether they had been harassed. The types of harassing experienced were varied with teasing reported as being most common. Other types of harassing behavior include insinuating comments, sexual overtures, and threats. She also stated covert and overt sexual harassment in almost all educational institutions exists in one-form male teachers. Female student have to put up with inappropriate behavior from male teachers. Liton, (2000) in 1999, the country was shocked when it was revealed that in JU (Jahanggirnagor University)' harassment of female students by other male students had reached horrendous proportions. A Fact Findings Committee by the Universities authorities revealed that more than 20 students of JU were raped and over 300 others sexually harassed on the campus. Ali (2006) stated that eve teasing is one of the worst forms of abuse form, which numerous women regardless of class, profession, and age are suffering. She also said eve teasing had created social insecurity for guardians of young girls and paved the way for dropout from schools, eventually leading to early marriage. Though eve teasing is a punishable offence in the Bangladesh Penal Code, it is hard for a victim to provide it in court.

Knowing the attitude towards eve teasing is important because the attitudes determine people's reaction and future course of action toward it. Present study is highly related to attitude of a person so we need to know about attitude. According to Krech, Crutchfield and Ballachey (1962) an individual attitude toward an object is a relatively enduring organization of beliefs and feelings about that object which predisposes her/him to respond in a certain way. An attitude is a hypothetical construct that represents an individual's degree of like or dislike for 
an item. Attitudes are generally positive or negative views of a person, place, thing, or event, this is often referred to as the attitude object. People can also be conflicted or ambivalent toward an object, meaning that they simultaneously possess both positive and negative attitudes toward the item in question. A positive attitude implies a way of thinking that is predominantly positive and optimistic. The opposite inclination, a negative attitude is predominantly pessimistic.

Today eve teasing is a typical crime. It is a traumatic experience, which can leave deep psychological scars and has negative consequence for the greater community. Most women in our society have been subjected to some form of sexual harassment as part of their daily. So eve teasing is more related to gender issue. Eve teasing may vary according to gender or level of education. However, in such incidents are very few. Now we need to know about 'Gender' and 'Level of education'. Gender refers to the sex of an individual, male or female, based on reproductive anatomy, and sexual identity, especially in relation to society or culture. In addition, level of education, which represents a broad section of the education "ladder" that is the progression from elementary to more complicated learning experience, embracing all fields and programmer groups that may occur at that particular stage of the progression.

Eve teasing is addressing to emerging issues. It has become a serious issues resulting in terrible and sometimes horrifying consequences. However, eve teasing is considered as a public nuisance all over the world, in our country it has gradually become brutal form of sexual harassment often resulting in grievous hurt, abduction, acid throwing, rape murder and forced suicide. Girls and women face rude comments, gesture with hands, legs or fingers and so on. In our country, there are so many loopholes and farces in the law that the perpetrators seldom are punished. As a result, the rate of eve teasing is increasing. Therefore, the present study is an attempt to explore the attitude of males and females of different educational level toward eve teasing which is expected that will help us to understand its causes, the current picture of eve teasing in our society. But unfortunately very few research has been done on this matter. Therefore, our present attempt is construct a scale that will help us to measure the attitude toward eve teasing. 
The hypotheses of the present study are

1) Attitude toward eve teasing will vary significantly according to gender (males and females) and

2) Eve teasing will vary according to levels of education (below primary 'one-four', five - S.S.C., H.S.C-Honors, and post-graduate).

\section{MATERIALS AND METHOD}

The purpose of the present study was to measure attitude toward eve teasing. For this purpose a scale for measuring attitude toward eve teasing was developed. However, the same sample was used to serve two purposes, for both the construction of the scale and to assess attitude toward eve teasing. The method of scale construction and measuring the attitude toward eve teasing according to sex, and education qualification are described below:

\section{Item Construction}

Initially, 40 Likert type statements related to the attitude toward eve teasing were constructed through survey of the related literature, opinion survey of students of different department of Dhaka University, internet, newspapers and case studies. Among these 40 were positive. These statements covered mainly the attitude toward eve teasing. To construct items, initially the discriminating characteristics and clarity of the items were considered carefully. After scrutinizing the initial statements, those were given to 8 teachers from the department of Psychology, Women and Gender Studies, Sociology, Social Welfare, Anthropology, public administration and Journalism to judge the statements according to their appropriateness to measure the attitude toward eve teasing. The judges were provided with an instruction to put their judgment on the 4-point scale provided with each statement. The four points ranged from 'strongly suitable to strongly unsuitable'. The judges were also requested to check up the statement, to revise or correct the statement, and suggest new statement if necessary. However, out of eight judges eight of them returned the questionnaire. Judges mean rating for each statement was used as a measure to weigh the statement on any of the four choices 1, 2, 3, and 4 rating points were assigned to consecutive choices. Here, the total score for each statement was determined by adding up all the corresponding rating values (from 1 to 4 ) for judges choices in a particular item. The maximum possible value could be 4 and the minimum possible value could be 1 . Adding the judges suggested items, the numbers of 
items became 29. For this study, "internal consistency" was used as the index of item validity or item analysis. Internal consistencies of items were calculated by applying Pearson's Product-Moment Correlation between the item scores and the total scores. The procedure of item analysis is describing as below.

\section{Sample}

The sample of the present research was consisted of 100 males and 100 females who were settled in two areas of Dhaka city named North Dhaka City Corporation and South Dhaka City Corporation. The data were collected by using random sampling. During the data collection, in South Dhaka City Corporation, I selected the Azimpur area, went to the Telephone Exchange Office, and collected the telephone number of local people. I wrote each of the number in white paper and kept it in a box. Then I drew lottery and selected 100 participants. In the same way, in the North Dhaka City Corporation I selected the area of Budda and went to the Telephone Exchange Office. I collected the total telephone number of local people of Budda area. I wrote each of the number in white paper and kept in a box. Then I drew lottery and selected 100 participants of Budda area. After that, I administered a questionnaire named attitude toward eve teasing on them. However all the respondents were literate adults who had different levels of education and were belonging to various socio-economic statuses. However, the same sample was used to serve two purposes for both the construction of the scale and to assess attitude toward eve teasing. The sample distribution is shown as follow:

TABLE 1: DISTRIBUTION OF STUDY SAMPLE ACCORDING TO LEVELS OF EDUCATION (BELOW PRIMARY 'ONE-FOUR', FIVE-S.S.C, H.S.CHONORS, AND POST GRADUATE).

\begin{tabular}{llllll}
\hline $\begin{array}{l}\text { Levels of } \\
\text { Education }\end{array}$ & One-four & Five - S.S.C & H.S.C- Honors & $\begin{array}{l}\text { Post } \\
\text { graduate }\end{array}$ & Total \\
Male & & & & & \\
Fender & 24 & 26 & 26 & 24 & 100 \\
Total & 24 & 26 & 26 & 24 & 100 \\
\hline
\end{tabular}




\section{Procedure}

The primarily selected 29 statements were arranged in a four-point scale (strongly agree to strongly disagree). The scale also consisted of a brief introduction and instruction of the test at its beginning and scope for respondents' comments and demographic data at its end. The statements were written one after another and in each statement, four boxes were provided for giving tick mark $(\sqrt{ })$ with each of the four choices in the four-point scale. The questionnaire was administered to each of the 200 respondents. The scores for positive item from 4 to 1 for "strongly agree" "agree" "disagree" "strongly disagree". Total score of the eve teasing for any individual was obtained from sum total scores on 29 items. The maximum possible score for this scale is 116 and the minimum possible score is 29 and the midpoint being 72.5. A lower total score indicates attitude toward eve teasing is higher. That means they are strongly in favor of eve teasing and higher total score indicates that they have less favorable attitude toward eve teasing.

\section{Item Analysis and Item Selection}

For item analysis, the total scores of the respondents were correlated with the total scores on each item obtained by all respondents. The Pearson ProductMoment Correlation method was employed for this purpose. Of the total 29 items, 10 were found to have significant item-total correlation ( $\alpha$ ranging from 0.01 to 0.05). And each of item is positive. These items were retained in the final scale.

TABLE 2 : THE FINAL SCALE FOE MEASURIING ATTITUDE TOWARD EVE TEASING.

\begin{tabular}{|c|c|c|}
\hline ক্রমিক নং & পদ সমূহ & সহসম্পর্ক (r) \\
\hline s & যাদের রুচিবোধ নিম্নমানের তারাই ইভটিজিং করে &.$- ১ ৭ 2^{*}$ \\
\hline ২ & নিজের শ্রেষ্টত্ব বলে জাহির করার একটা উপায় হল ইভটিজিং করা &.$৩ ০ ৩ * *$ \\
\hline ৩ & পাশচাত্য সংস্কৃতিকে অন্ধভাবে অনুকরণ করার কারণে ইভটিজিং হচ্ছে & .২০৩** \\
\hline 8 & যৌন চাহিদাকে পরোক্ষভাবে পূরণের একটা মাধ্যম হিলেবে ইভটিজিং কাজ করে & .১৬৯* \\
\hline$\varangle$ & কেবল কৌতুহলী বশে ইভটিজিং করে & .১११* \\
\hline 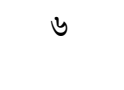 & $\begin{array}{l}\text { মহিলাদের প্রতি পুরুষদের যে একটা আক্রমনাত্বক মনোভাব রয়েছে তা ইভটিজিং এর মাধ্যমে } \\
\text { প্রকাশ পায় }\end{array}$ &.$২ ০ ৩ * *$ \\
\hline १ & অন্যদেরকে অনুকরণ করে অনেকে ইভটিজিং করে &.$১ ৬ ৫ *$ \\
\hline$b$ & কোন নারীর প্রতি যে অব্যক্ত বাসনা রয়েছে তা ইভটিজিং এর মাধ্যনে প্রকাশ পায় & ৩২২** \\
\hline$৯$ & অনেক সময় ব্যক্তি যাকে পছন্দ করে সে যদি তাকে পাত্তা না দেয় তখন সে ইভটিজিং করে & $. \Delta b-q * *$ \\
\hline ১০ & নারীদের প্রতি অবদমিত যৌন চাহিদাকে প্রকাশের অন্যতম মাধ্যম হল ইভটিজিং করা & .২১৯** \\
\hline
\end{tabular}

* Significant at $=.05$

$* *$ Significant at $=.01$ 
BIPLOB KUMAR DEY et al.

Determination of reliability and validity of the scale

\section{Reliability}

There are various methods of estimating reliability of a scale. Spilt-half and Chronbach's alpha were computed as measure of reliability. In Spilt-half reliability was assessed by splitting the scale into two halves, using even-odd method. After splitting the scale into two halves, total scores were computed and the correlation (r) between the two parts was calculated. The spilt-half reliability of this scale was 0.52 which was significant at $\alpha=0.001$ and in Chronbach,s alpha was computed as a measure of reliability which was 0.56 for this scale and was significant.

\section{Validity}

The face validity of the scale was checked by 8 judges who were teachers from the department of Psychology, Women and Gender Studies, Sociology, Social Welfare, Anthropology, public administration and Journalism of University of Dhaka. As mentioned earlier all items retained in the scale were judged by experts. Futhermore, the scale can be expected valid because it constituted only the valid items (Anastasi 1982).

\section{RESULTS AND DISCUSSION}

The present study was conducted to see the attitude toward eve teasing. The obtained were analyzed by using a 2-way ANOVA. The mean scores of attitude toward eve teasing according to gender and levels of education are presented in table 3 and the results of 2-way ANOVA are presented in table 4 respectively. 
TABLE 3: THE MEAN SCORE AND STANDARD DEVIATION OF EVE TEASING AS A FUNCTION OF GENDER AND LEVELS OF EDUCATION.

\begin{tabular}{|c|c|c|c|c|}
\hline Gender & Level of Education & Mean & Std. Deviation & $\mathrm{N}$ \\
\hline \multirow[t]{5}{*}{ Male } & Below primary(one-four) & 27.1667 & 3.08808 & 24 \\
\hline & Secondary(five-s.s.c.) & 25.1923 & 3.21272 & 26 \\
\hline & H.S.C.-Honours & 26.8462 & 3.81253 & 26 \\
\hline & Post graduate & 25.0833 & 3.29580 & 24 \\
\hline & Total & 26.0700 & 3.45024 & 100 \\
\hline \multirow[t]{5}{*}{ Female } & Selow primary(one-four) & 25.0417 & 2.56191 & 24 \\
\hline & Secondary(five-s.s.c.) & 27.3077 & 4.06713 & 26 \\
\hline & H.S.C.-Honours & 27.1923 & 4.23338 & 26 \\
\hline & Post graduate & 27.9583 & 4.47679 & 24 \\
\hline & Total & 26.8900 & 4.00730 & 100 \\
\hline \multirow[t]{4}{*}{ Total } & Below primary(one-four) & 26.1042 & 3.00524 & 48 \\
\hline & Secondary(five-s.s.c.) & 26.2500 & 3.78270 & 52 \\
\hline & H.S.C.-Honours & 27.0192 & 3.99259 & 52 \\
\hline & Post graduate & 26.5208 & 4.15134 & 48 \\
\hline
\end{tabular}

From table 3 it is seen that males whose education level is below primary scored $(\bar{x}=27.17)$ compared to female $(\bar{x}=25.04)$, In case of class five to S.S.C. males scored $\bar{x}=25.19$ and females scored $\bar{x}=27.31$ respectively. Male whose education level is H.S.C.to Honors scored $\bar{x}=26.85$ and females scored $\bar{x}=27.19$. Finally postgraduate male scored is $\bar{x}=25.08$ and female scored 27.96 toward eve teasing. It should be mentioned here that the midpoint of the eve teasing scale is 25 and it seems that male attitude to eve teasing is higher than that of females except in case of those with primary education. 
BIPLOB KUMAR DEY et al.

TABLE 4: ANOVA OF EVE TEASING SCORES AS A FUNCTION OF GENDER AND EDUCATION.

\begin{tabular}{lllllll}
\hline Source & $\begin{array}{l}\text { Type III Sum } \\
\text { of Squares }\end{array}$ & df & Mean Square & F & Sig. \\
& & & & & & \\
& & 1 & 32.180 & 2.410 & 0.122 \\
Gender & 32.180 & 3 & 8.244 & 0.617 & 0.605 \\
Education & 24.731 & 3 & & 59.829 & 4.480 & 0.005 \\
Gender * Education & 179.486 & 3 & & & \\
Error & 2564.083 & 192 & 13.355 & & \\
Total & 143040.00 & & 200 & & &
\end{tabular}

It is seen from table 4 that the main effect of gender toward the attitude of eve teasing is non-significant $(\mathrm{F}=2.410, \mathrm{df}=1,192)$. That means the attitude toward the eve teasing did not vary significantly according to gender. Table 3 shows that the mean scores of eve teasing of males were $\bar{x}=26.07$ and females were $\bar{x}=26.89$ respectively. It is seen from table 3 that the main effect of levels of education toward the attitude of eve teasing is non-significant $(\mathrm{F}=.617, \mathrm{df}=3,192)$. That means the attitude toward the eve teasing did not vary significantly according to levels of education. Table 3 shows that the means scores of eve teasing of below primary (one-four) $\bar{x}=26.10$; secondary (five-S.S.C) $\bar{x}=26.25$; H.S.C.-Honors $\bar{x}=27.019$ and postgraduate $\bar{x}=26.52$ respectively. However the table 4 it was seen that the interaction effect toward eve teasing was significantly found $(\mathrm{F}=4.48, \mathrm{df}=3,192 ;<.005)$.

The main finding of the present study is that, eve teasing does not vary according to gender. Male and female are now conducting eve teasing. At present developing of civilization, are entering into different work place with male competitor. Therefore, they are modern and conscious about their life style. They are now enjoying equal opportunity in every sphere of life. So, women are practicing the unwanted behavior like eve teasing. In past, men were practicing eve teasing but in the present time, both of them are conducting eve teasing. 
Eve teasing does not vary according to level of education, because, our social system is now gradually improving. We always give effort to discover something by eliminating social problem. At present, a radical change has occurred in our education system. Now, most people are educated and they are achieving their qualification or knowledge from different educational institutions in our country or abroad. However, most of time they only gather certificate and traditional knowledge. As a result, their ethics, value, attitude have been demoralizing. So there are many unusual event like eve teasing have been occurred by students of different level of education system. Many students are watching cinema, motion picture, and imitating western culture and trying to present as a hero among their friends circle by conducting unwanted behavior like eve teasing. On the other hand, some illiterate or low educated people are conducting eve teasing by imitating this unwanted behavior. So eve teasing does not vary according to level of education.

However, the interaction effect between gender and level of education are significant. Actually, eve teasing does not vary particularly with gender and level of education but only vary in their interaction effect. Those females who are illiterate or bellow primary, they stay inside the home; they contract less interaction with social situation. As a result, they are unaware about their life and society. So, they do not face and realize the problem (eve teasing) than literate female. As a result, their scores are lower than that of literate girls. Sometimes their illiteracy leads to a girl early marriage.

Now a day's literate female has a better position than the illiterate female and they are contributing to the national developing process. At present, they are going to school, college, and universities for expecting better life. However, it is very sorrowful matter that sometimes they have to face various types of sexual harassment, eve teasing, vulgar comments and it creates social insecurity for guardians of young girls and paves the way for dropout from school eventually leading to marriage and (Ali 2006) found same result from his studying.

In the present world, females are involved various developmental activities, they are going outside the home, and entering the industries, higher educational institutions, factories and companies. That means, their rate of interaction is increasing. As a result, they fall victims to the male people. Sometimes girls are found covert \& overt sexual harassment in educational institution by male teachers. Results of the present study are similar to those of Huda (1999). On the other hand, male does not accept the women's empowerment $\&$ development. Therefore, they are falling harassment or eve teasing and their score is higher than the illiterate girls or the girls who are in bellow primary. 
The males of all category of education level show their positive attitude toward eve teasing. Actually, males are conducting these unwanted events but sometimes they confess it or sometimes they do not confess it. Some male realizes that it is a problem but their number is very few. Actually, most of the time, they do not feel it. So, their scores are lower than girls.

The main limitation of this present study is that some of the respondents did not provide actual attitude about eve teasing because eve teasing is a very sensitive issue. Sometimes respondents felt fear about completion of the questionnaire, although a rapport was established and maintained confidentiality. So, collecting of data was troublesome.

It may be concluded from the above discussion that laws dealing with eve teasing should be implemented properly and patriarchy should be eliminated from the society. Culprits should be punished strictly and the victims of eve teasing must be encouraged to protest against the offence and media should be used to promote more positive images of women and practice culture against eve teasing.

\section{REFERENCES}

ALI, S.2006. Bangladesh National Women Lawyer Association to launch

Campaign against hecklers of women. The New Age, May 7, pp. 61-78.

ANASTASI, J.R. L.R.1982. Psychological testing and Assessment $5^{\text {th }}$ Ed., New York: Macmillan Publishing Co. INC.pp.106-107.

BERRET, G.2006. Eve Teasing. The Official Dictionary of unofficial English. New York: McGraw-Hill Professional. 106 pp.

CEC, 1993. Sexual Harassment at work place in the European Union, Luxemberg and Office for Officials Publications of the European Communities. The official journal of European communities, 20:37.

HUDA, S. 1999. Perspectives on Sexual Harassment in Bangladesh: Acknowledge its Experience, A Journal of Women for Women, 6:19-28.

KRECH, D., CRUTCHFIELD, R.S. AND BALLACHEY,E.L.1962. Individual in Society. New York: McGraw Hill.pp.339-380.

Liton, S. 2000. The Daily Star. September 26.

PURI, J. 1999. Woman, body, desire in post-Colonial India: Narratives of Gender and Sexuality. Routledge. $87 \mathrm{pp}$.

SHAMPA, F.R.2003. The young girls killed themselves after sexual harassment. The Star Weekend Magazine August 17, pp. 27-36.

Manuscript received on 24.12.2011; Accepted on 07.07.2012

The Chittagong University Journal of Biological Sciences, Vol. 6 (1 \& 2). Page No. 